\title{
Bone mineral density and fracture risk in adult patients with hypophosphatasia
}

\author{
F. Genest ${ }^{1} \cdot$ L. Claußen ${ }^{1} \cdot$ D. Rak ${ }^{1} \cdot$ L. Seefried ${ }^{1}$ (B)
}

Received: 29 April 2020 / Accepted: 25 August 2020 / Published online: 2 September 2020

(C) The Author(s) 2020

\begin{abstract}
Summary In adult hypophosphatasia (HPP) patients, elevated lumbar spine dual X-ray absorptiometry (DXA) values are associated with markers of disease severity and disease-specific fracture risk while femoral bone mineral density (BMD), being largely unaffected by the disease severity, may still be useful to monitor other causes of increased fracture risk due to low BMD. Introduction Hypophosphatasia (HPP) is a rare inherited metabolic disorder due to deficient activity of the tissue-nonspecific alkaline phosphatase (TNAP). Clinical manifestation in adult HPP patients is manifold including an increased risk for fractures, but data regarding clinical significance of DXA measurement and associations with fracture risk and disease severity is scarce. Methods Retrospective single-center analysis of DXA scans in patients with confirmed HPP (documented mutation, clinical symptoms, low alkaline phosphatase activity). Further data evaluation included disease-related fractures, laboratory results (alkaline phosphatase, pyridoxalphosphate, phosphoethanolamine), and medical history.

Results Analysis included 110 patients ( 84 female, mean age of 46.2 years) of whom $37.3 \%(n=41)$ were harboring two mutations. Average T-Score level at the lumbar spine was - 0.1 (SD 1.9), and mean total hip T-Score was - 1.07 (SD 0.15). Both lower ALP activity and higher substrate levels (pyridoxalphosphate and phosphoethanolamine) were significantly correlated with increased lumbar spine T-Score levels $(p<0.001)$ while BMD at the hip was not affected by indicators of disease severity. Increased lumbar spine BMD was significantly associated with an increased risk for HPP-related fractures, prevalent in $22(20 \%)$ patients $(p<0.001)$ with 21 of them having biallelic mutations.

Conclusion BMD in adult HPP patients is not systematically reduced. Conversely, increased lumbar spine BMD appears to be associated with severely compromised mineralization and increased risk for HPP-related fractures while BMD at the hip appears unaffected by indicators of disease severity, suggesting suitability of this anatomic location for assessing and discerning disorders with increased fracture risk owing to reduced BMD like osteoporosis.
\end{abstract}

Trial registration number German register for clinical studies (DRKS00014022)

Date of registration $02 / 10 / 2018$ - retrospectively registered

Keywords Bone mineral density $\cdot$ Fracture risk $\cdot$ Hypophosphatasia $\cdot$ Osteoporosis $\cdot$ Pseudofracture

\section{Introduction}

Hypophosphatasia (HPP) is a rare genetic disorder caused by pathogenic variants in the ALPL gene entailing deficient activity of the tissue-nonspecific alkaline phosphatase (TNAP)

Electronic supplementary material The online version of this article (https://doi.org/10.1007/s00198-020-05612-9) contains supplementary material, which is available to authorized users.

L. Seefried

l-seefried.klh@uni-wuerzburg.de

1 Clinical Trial Unit, Orthopedic Department, University of Wuerzburg, Brettreichstrasse 11, 97074 Wuerzburg, Germany
[1]. Clinical manifestation of the disorder comprises a wide spectrum of severity, ranging from life-threatening disease in infants to largely asymptomatic carriers [2-4]. Particularly in adult patients, the phenotypic presentation is manifold, including bone issues with fractures, bone marrow lesions and musculoskeletal pain, and exhaustion and fatigue as well as supposed gastrointestinal and neuro-psychiatric symptoms $[1,5$, 6]. While the underlying pathophysiologic mechanisms for many of these symptoms still have to be elucidated in detail, defective bone mineralization in HPP is well recognized [7]. Increased fracture rate and a high prevalence of insufficiency fractures and pseudofractures as well as recurrent bone marrow lesions are considered a hallmark of HPP in adult patients $[4,8-10]$. However, this does not apply to all patients and by 
now, there is no diagnostic marker or tool to anticipate what HPP patients are at risk for fractures. Furthermore, thanks to growing awareness for HPP among health care professionals, heterozygous ALPL variants are increasingly detected during routine diagnostic workup of osteoporosis and osteoporotic fractures, evoking the question if and how such an ALPL variant affects bone mineral density and appropriate clinical management $[11,12]$.

Assessing bone mineral density (BMD) by means of dual X-ray absorptiometry (DXA) is well established and standard of care for evaluating fracture risk in osteoporosis $[13,14]$ but only few studies with small case numbers provide data on BMD in adult patients with HPP $[4,6,8,15,16]$. Reported findings are heterogeneous and have not been associated with fracture pattern or prevalence, yet. Hence, it is unknown if BMD in adult patients with HPP is altered consistently and to what extent BMD measurement alone or in combination with other well-established parameters of the disease like ALP, pyridoxalphosphate (PLP), and phosphoethanolamine (PEA) can contribute to understanding and predicting fracture risk in HPP.

Considering differential localization and morphology of fractures observed in patients with adult HPP, there is a distinct type of fracture with supposedly different underlying pathophysiologic mechanisms that can readily be distinguished from, e.g., osteoporotic fractures. Pseudofractures (syn. Looser Zones), i.e., atraumatic radiolucencies, perpendicular to the cortex, incompletely spanning the diameter of the bone and affecting only one cortex on plain x-ray films as well as complete insufficiency fractures, i.e., atraumatic lucencies extending across both cortices, can be considered a hallmark of deficient mineralization and impaired remodeling. Owing to a supposedly insufficient healing response, these areas can exhibit cortical beaking or flaring. Depending on the individual situation, these lesions can finally heal, reoccur, or progress and eventually fracture completely upon minimal trauma. In HPP, pseudofractures most frequently occur along the diaphyseal axis of the weight-bearing lower extremities, specifically the femur $[17,18]$ and the metatarsal bones but can also be observed at other bones [1, 19, 20].

Despite continuously growing awareness for HPP both scientifically and in daily clinical routine, there are still no diagnostic landmarks helping to identify HPP patients at risk for developing any of these fractures, even though this surely is a key determinant to guide individual treatment strategies.

Combining BMD data and clinical information consistently obtained from a large cohort of adult HPP patients, this evaluation aims at elucidating a consistent pattern of BMD alterations in HPP patients and identifying to what extent and in combination with what other risk factors this may be helpful to anticipate the risk for specific fractures in HPP patients.

\section{Material and methods}

\section{Study design and patients}

This is a retrospective, single-center analysis of medical records of adult patients with HPP. The evaluation is based on the results from BMD assessments by DXA, medical history, and course of the disease including fracture history and laboratory evaluations, specifically markers of bone and mineral metabolism (ALP, serum-phosphorus, NTX, vitamin D) and disease-specific substrate levels (PLP, PEA). For consistency, only datasets of patients meeting the following selection criteria were included:

- Confirmed pathogenic variant within the ALPL gene

- HPP-related clinical signs and symptoms

- At least one of the following laboratory findings:

- Serum ALP levels below age- and gender-adjusted lower normal range (at least two measurements with an interval of $>4$ weeks)

- Serum PLP and/or urinary PEA above upper normal limit

- DXA scan performed on-site with the same Dual X-Ray Absorptiometry Scanner (Hologic Discovery A)

- No previous or current enzyme replacement therapy with Asfotase alfa at the time of the DXA scan

Searching the on-site medical database, 110 HPP patients meeting the above criteria were identified. For those patients, information on medical history specifically fracture history and fracture types along with lab results as specified above were extracted from the medical records and the imaging database. Evaluation of medical records specifically included information on HPP-related childhood symptoms, particularly craniosynostosis, scoliosis, bone deformities, delayed gross motor development, premature loss of primary teeth before 5 years of age, and loss of primary teeth with root still attached.

In order to assess disease-associated fracture risk, a subgroup analysis was conducted including those patients who had experienced an HPP-related fracture without causative trauma. However, sometimes minimal trauma or repetitive strain is associated with the fracture becoming symptomatic for the first time. Shortly, fractures in adulthood were categorized as being HPP-related if two or more of the criteria specified below [21, 22]. Fractures due to appropriate trauma, childhood fractures, and bone marrow lesions were not included in this analysis.

In order to be termed HPP-related, fractures had to meet at least two of the following criteria:

- No/minimal trauma

- Pseudofracture/bicortical lucency 
- Beaking/flaring

- Prodromal pain

- Diaphyseal fracture

All DXA scans were obtained using the same scanner (Hologic Discovery A). If not precluded by metal implants, T-Scores and Z-Scores were obtained both at the lumbar spine and bilaterally at the proximal femur, evaluating both femoral neck and total femur. As for the femoral results, the side with the lowest value was used for further analysis. In the case of metal implants or total arthroplasty of the hip on one side, the contralateral side was measured and used for analysis. For the spine measurement, average result of lumbar vertebral bodies L1-4 was used for calculating T- and Z-Scores. Vertebrae yielding invalid results due to deformities, severe osteochondrosis, or other artifacts, i.e., deformities, implants, cement-augmentation, were excluded from the analysis and average value of the remaining intact lumbar vertebral bodies was analyzed. Lumbar spine BMD was considered invalid and not included in the analysis if less than two vertebrae were assessable.

For consistency, lab results for ALP, serum-phosphorus, NTX, and vitamin D considered in the analysis were those obtained on-site at the same date as the DXA scan was performed and analyzed in the same laboratory using the same assays to reduce inter-assay variability. Quantitation of PLP was performed using serum samples, and PEA normalized to creatinine excretion was determined from urine samples.

\section{Statistics}

Statistical analysis comprised absolute frequencies, proportions, and arithmetic means. Correlations were calculated using Pearson's correlation coefficient and $\chi^{2}$ test. Differences were assessed using independent samples $t$ test. $P$ values of less than 0.05 were considered statistically significant. All statistical analyses and figures were calculated using SPSS ver. 25 statistical software package (IBM Corp. Released 2017. IBM SPSS Statistics for Windows, Version 25.0. Armonk, NY: IBM Corp.). The study design was reviewed by the ethics committee of the University of Wuerzburg (Nr. 20180105 01) and registered with the German Clinical Trials Register (DRKS00014022).

\section{Results}

Out of 110 patients identified meeting the above selection criteria, 84 (76.4\%) were female and 26 (23.6\%) were male. All patients had genetically confirmed variants in the ALPL gene with $69(62.7 \%)$ being heterozygously affected and 41 (37.3\%) having two mutations (39 compound heterozygous, 2 homozygous). While both these groups were well balanced with regard to age and constitutional parameters, there were apparent differences regarding disease-specific laboratory parameters with significantly lower ALP activity and higher substrate levels for PLP and urinary PEA as well as higher serum-phosphorus in those subjects with two mutations. Baseline characteristics are detailed in Table 1.

\section{Bone mineral density}

Mean lumbar spine T-Score and Z-Score in the overall cohort were -0.09 (SD 1.88; range -3.2 to +7.0$)$ and +0.55 (SD $2.03 ;-3.1$ to +7.5$)$, respectively. For the total femur, average T-Score was -1.07 (SD 1.16; -3.4 to +3.7 ) and Z-Score was -0.56 (SD $1.19 ;-3.0$ to +3.9 ). At the femoral neck, the TScore was -1.73 (SD 1.24; -4.3 to +3.3 ) and the Z-Score was -0.85 (SD 1.24; -3.9 to +3.8$)$. Both lumbar spine ZScore $(p=0.001)$ and total femur Z-Score $(p=0.023)$ increased significantly with progressive aging (see Fig. 1a/b). There were no significant correlations between age and lumbar spine T-Score $(p=0.522)$ or femoral T-Score values ( $p=$ $0.195)$. The femoral neck T-Score $(p=0.013)$ as well as $Z$ Score $(p=0.015)$ showed significant correlations with age. In general, there was a tendency for increasing BMD with progressive age, particularly at the lumbar spine.

Furthermore, lumbar spine T-Score showed a significant negative correlation with ALP levels $(p=0.018)$, i.e., lower ALP levels were associated with higher lumbar spine TScores, whereas femoral T-Score was not significantly correlated with ALP levels (Fig. 2a/b).

Comparing male with female patients, there were no significant differences regarding BMD T- and Z-Scores neither at the lumbar spine nor at the femur. Analyzing BMD data for patients with one or two pathogenic variants, there were no statistically significant differences at the femoral sites, neither at the femoral neck nor at the total femur. However, there was a statistically significant difference of BMD values between these groups at the lumbar spine with essentially normal ageadjusted values for heterozygously affected patients (average Z-Score - 0.12) while patients with two genetic variants exhibited significantly higher lumbar spine BMD (Z-Score + 1.71) (Fig. 3) (for detailed comparisons, see also Table 2).

\section{Fractures}

In total, 53 out of the 110 HPP patients (48.2\%) had already experienced at least one fracture over lifetime. However, fractures without or due to minimal/insufficient trauma were documented in only 31 patients $(28.2 \%)$. Out of these, 22 patients $(20 \%)$ exhibited HPP-related fractures according to the criteria outlined above while the other fractures appeared to be rather osteoporotic. HPP-related fractures were most prevalent in the subtrochanteric region of the femur ( $n=15$ patients), the metatarsal bones ( $n=13$ patients), and the tibia ( $n=6$ patients) and affected preferentially 
Table 1 Overview for anthropometrics, lab values, and BMD for all patients and gender dependent

\begin{tabular}{lllll}
\hline Mean (SD) & $\begin{array}{l}\text { All patients } \\
(n=110)\end{array}$ & $\begin{array}{l}\text { Female patients } \\
(n=84 / 76.4 \%)\end{array}$ & $\begin{array}{l}\text { Male patients } \\
(n=26 / 23.6 \%)\end{array}$ & $p$ value* \\
\hline Anthropometrics & & & & \\
Age (year) & $46.20(13.8)$ & $46.23(14.0)$ & $46.12(13.6)$ & 0.972 \\
Height (cm) & $167.33(9.8)$ & $166.37(9.5)$ & $170.42(10.6)$ & 0.066 \\
Weight (kg) & $72.95(16.9)$ & $74.18(17.3)$ & $69.00(15.0)$ & 0.173 \\
BMI (kg/m $\left.{ }^{2}\right)$ & $25.98(5.4)$ & $26.74(5.7)$ & $23.54(3.3)$ & $0.007^{*}$ \\
Lab values (normal range) & & & & \\
ALP (f: 42-98/m: 53-128 U/l) & $24.39(11.6)$ & $24.42(11.7)$ & $24.31(11.6)$ & 0.967 \\
Phosphate (0.6-1.8 mmol/l) & $1.36(0.2)$ & $1.38(0.2)$ & $1.28(0.2)$ & 0.068 \\
PLP (5-30 ng/ml) & $154.25(281.2)$ & $157.42(303.1)$ & $143.18(190.3)$ & 0.828 \\
uPEA (2.3-11.3 mmol/mol Krea) & $25.43(33.4)$ & $25.76(36.3)$ & $24.35(21.9)$ & 0.857 \\
NTX (12.9-22.7 nM BCE/l) & $13.48(8.1)$ & $13.64(9.0)$ & $12.96(3.6)$ & 0.616 \\
Vitamin D (20-40 ng/ml) & $25.51(12.6)$ & $25.44(12.5)$ & $25.73(13.2)$ & 0.927 \\
BMD & & & & \\
Lumbar spine T-Score & $-0.09(1.9)$ & $+0.02(1.8)$ & $-0.48(2.0)$ & 0.247 \\
Lumbar spine Z-Score & $+0.55(2.0)$ & $+0.75(2.0)$ & $-0.11(2.0)$ & 0.061 \\
Total femur T-Score & $-1.07(1.2)$ & $-1.12(1.1)$ & $-0.92(1.2)$ & 0.455 \\
Total femur Z-Score & $-0.56(1.2)$ & $-0.64(1.2)$ & $-0.32(1.2)$ & 0.237 \\
Femoral neck T-Score & $-1.73(1.2)$ & $-1.69(1.3)$ & $-1.86(1.2)$ & 0.556 \\
Femoral neck Z-Score & $-0.85(1.2)$ & $-0.87(1.3)$ & $-0.78(1.1)$ & 0.740 \\
\hline
\end{tabular}

*Significant differences between female and male patients
(21 out of the 22) compound heterozygous/homozygous patients and only one heterozygous patient.

Patients with specific HPP-associated fractures were sig. older $(p=0.013)$ than the rest, but did not show any significant difference in terms of their physical constitution otherwise. However, patients with HPP-related fractures had significantly lower ALP activity $(p<0.001)$ and elevated substrate levels for PLP ( $p=$ $0.001)$ and urinary PEA $(p=0.001)$ along with higher blood levels for serum-phosphorus $(p<0.001)$ than other patients. In addition, lumbar spine T- and Z-Scores were significantly higher ( $p<0.001$ for both) in patients with HPP-specific fractures, while femoral T- and Z-Scores did not exhibit any significant difference In 19 out of the 22 patients with fractures, lumbar spine ZScore was $\geq 0.0$ and never below -1.0 in any patient. Accordingly, while there was a significant correlation of total hip and lumbar spine T-Score results in those patients without HPP-related fractures $(r=0.715, p<0.001)$, in those patients with HPP-related fractures, high lumbar spine T-Score results did not correlate with the femoral values $(r=0.196, p=0.564)$. A detailed comparison of patient characteristics with regard to the subgroups of patients with and without HPP-related fractures is provided in Table 3.

\section{Patients with risk for an HPP-related fracture}

In order to identify risk factors for HPP-related fractures, correlations between patients with HPP-specific fractures and other HPP characteristic parameters and symptoms were analyzed. There were significant correlations between HPPspecific fractures and having two variants in the ALPL gene $(p<0.001)$, low ALPL levels below $20 \mathrm{U} / 1(p=0.001)$, elevated serum phosphorous $>1.5 \mathrm{mmol} / 1(p<0.001)$, and elevated PLP $>30 \mathrm{ng} / \mathrm{ml}(p=0.001)$. In addition, elevated lumbar spine $\mathrm{T}$-Score $\geq 0$ was significantly correlated with the prevalence of HPP-related fractures $(p=0.003)$. Conversely, there were no significant correlations of HPP-related fractures with reduced femoral or lumbar spine T-Scores $\leq-2.5$ ( $p=$ $0.203 ; p=0.524)$. Low vitamin D levels $(p=0.131)$, as well as elevated NTX $(p=0.433)$, did not exhibit any association with HPP-related fracture risk.

\section{Discussion}

The objective of this study was to evaluate BMD patterns detected with DXA in adult HPP patients in order to assess if DXA findings and other hallmarks of the disease are suitable to indicate the risk for HPP-related fractures. In order to have a consistent dataset, only patients with a documented genetic variant, low ALP, and both BMD assessments and laboratory workup performed with the same devices and tools at the same institution were included in this analysis.

Overall, BMD was not generally decreased in these HPP patients and particularly lumbar spine T- and Z-Scores were 
Fig. 1 Association between a lumbar Z-Score and age and $\mathbf{b}$ femoral Z-Score and age
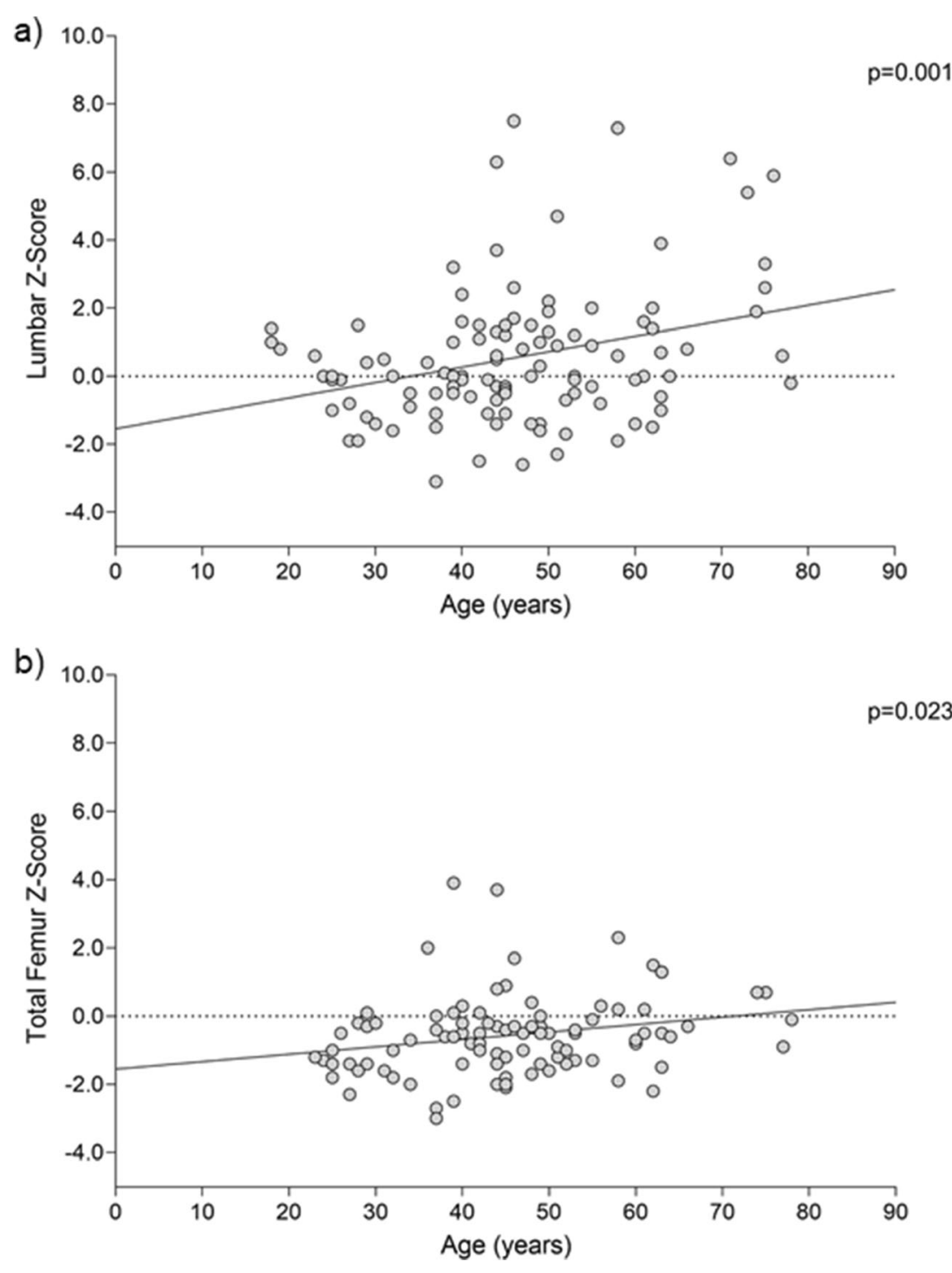

rather increased. Specifically, patients with two mutations, lower ALP levels, and elevated levels for serum-phosphorus, PLP, and urinary PEA, i.e., most severely affected individuals, were showing significantly higher lumbar spine T- and ZScores. Moreover, lumbar spine BMD increased with progressive aging. A potential explanation for this finding of high lumbar spine BMD in more severely affected patients and with progressive aging could be compensatory appositional bone formation resembling a diffuse idiopathic skeletal hyperostosis and heterotopic calcification of neighboring soft tissue structures and ligaments as well as supposed osteoid overload potentially combined with structurally inappropriate mineralization [8, 23-26]. The concept of such qualitative compromise of bone in the most severely affected patients is supported by the finding of these increased lumbar T- and Z-Scores being associated with HPP-related fractures. Even though the majority of HPP-related fractures were observed along the lower extremities, i.e., femur, metatarsal bones, and tibia, femoral BMD was not significantly altered and not a valid prognostic marker for this kind of fracture. This divergence is further substantiated by the finding that in those patients with HPP-related fractures, we could not confirm the generally well-established correlation of lumbar spine and total hip BMD values [27].

While accumulation of inorganic pyrophosphate (PPi) in HPP is considered to compromise proper bone mineralization, extracellular accumulation of PPi in soft tissues is supposed to trigger calcium pyrophosphate dihydrate (CPPD) deposition and crystal formation near joints and calcification of ligaments [24, 26, 28, 29]. Accordingly, inappropriately high lumbar spine DXA values due to, e.g., heterotopic soft tissue calcifications around the spine may thus be an indicator of particularly high PPi load which on the other hand is also associated with severely compromised bone mineralization, predisposing to pseudofractures. Since valid assays for direct measurement of PPi load are not available in clinical routine, yet, 
Fig. 2 Correlation between a lumbar spine T-Score and ALPlevels and $\mathbf{b}$ femoral T-Score and ALP levels

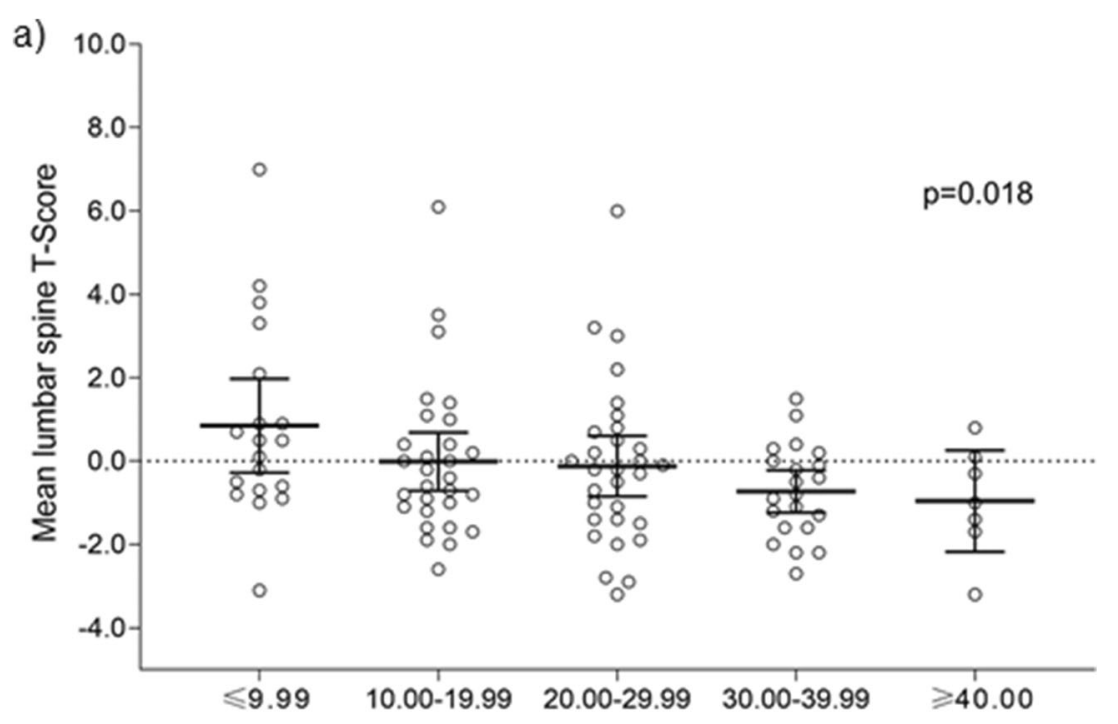

ALP Subgroups (U/l)

b)

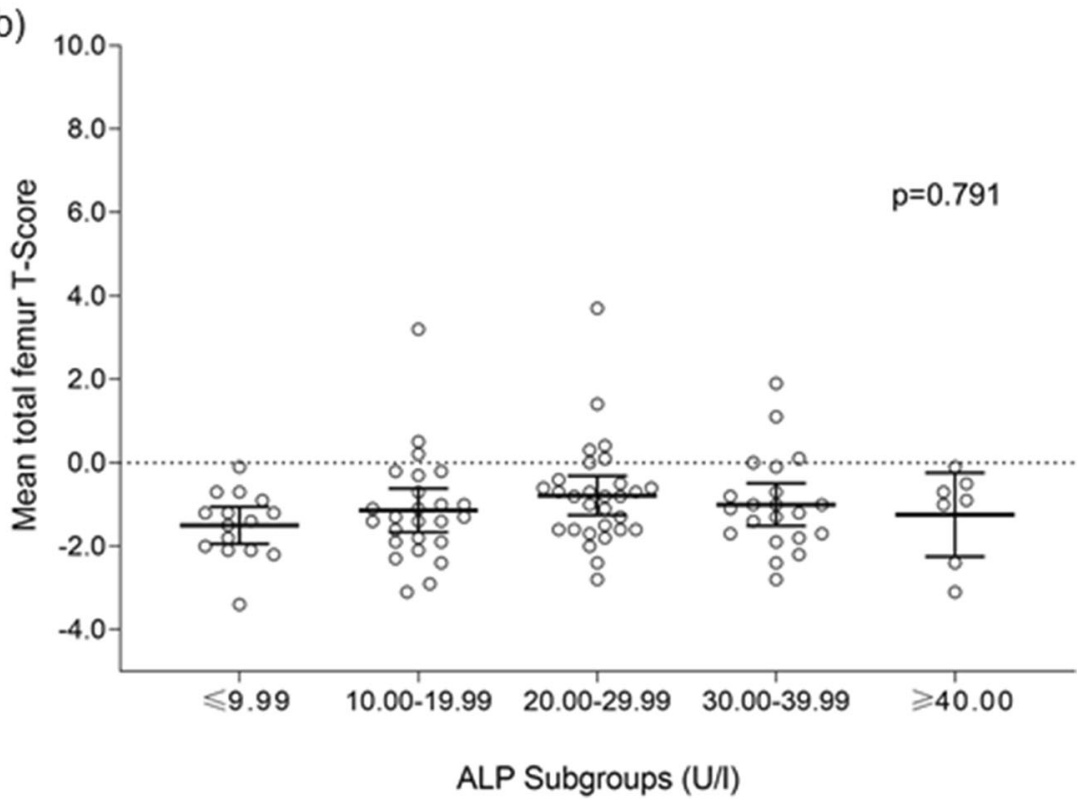

understanding this association appears even more important in order to determine an individual patient's specific fracture risk. This actually corresponds to the finding of patients with lower ALP activity having significantly higher lumbar spine BMD (Fig. 2a).

Conversely, femoral BMD did not correlate with residual ALP activity, and femoral results did not significantly differ between patients with 1 vs. 2 mutations and patients with vs. those without HPP-related fractures suggesting that with regard to osteoporosis testing in patients with ALPL variants, specifically at higher age, this anatomic region may be more suitable and meaningful for assessing osteoporosis-related fracture risk.
It surely would have been appealing to make a direct comparison of HPP-related and osteoporotic fractures in HPP patients but at this point, the number of attributable osteoporotic fractures in this mixed cohort was too small to draw robust and clinically meaningful conclusion. Indeed a preliminary review of individual cases (data not shown) with osteoporotic fractures suggests less reduced ALP levels and less elevated serum-phosphorus, PLP, and urinary PEA levels along with reduced femoral BMD as indicators for osteoporotic fractures.

The findings of our study confirm and substantiate what has been expert-perceived knowledge before: Disease-related fracture risk in adult patients with HPP exhibits a distinctive pattern as compared with other conditions with increased risk 
Table 2 Comparison of patients with 1 or 2 ALPL variants regarding laboratory results and BMD

\begin{tabular}{|c|c|c|c|}
\hline Mean (SD) & $\begin{array}{l}\text { Patients with } 1 \text { variant } \\
(n=69 / 62.7 \%)\end{array}$ & $\begin{array}{l}\text { Patients with } 2 \text { variants } \\
(n=41 / 37.3 \%)\end{array}$ & $p$ value* \\
\hline \multicolumn{4}{|l|}{ Anthropometrics } \\
\hline Age (year) & $44.32(12.0)$ & $49.37(16.1)$ & 0.086 \\
\hline Height (cm) & $167.43(9.5)$ & $167.15(10.5)$ & 0.883 \\
\hline Weight $(\mathrm{kg})$ & $72.22(17.2)$ & $74.20(16.5)$ & 0.555 \\
\hline BMI $\left(\mathrm{kg} / \mathrm{m}^{2}\right)$ & $25.74(5.9)$ & $26.39(4.4)$ & 0.545 \\
\hline \multicolumn{4}{|l|}{ Lab values (normal range) } \\
\hline ALP (f: 42-98/m: 53-128 U/l) & $28.93(10.0)$ & $16.76(10.2)$ & $<0.001^{*}$ \\
\hline Phosphate $(0.6-1.8 \mathrm{mmol} / \mathrm{l})$ & $1.26(0.2)$ & $1.53(0.2)$ & $<0.001^{*}$ \\
\hline PLP (5-30 ng/ml) & $47.42(32.7)$ & $343.26(404.1)$ & $<0.001^{*}$ \\
\hline uPEA (2.3-11.3 mmol/mol Krea) & $12.24(12.2)$ & $47.64(44.5)$ & $<0.001 *$ \\
\hline NTX (12.9-22.7 nM BCE/1) & $12.57(4.5)$ & $15.01(11.4)$ & $0.037 *$ \\
\hline Vitamin D (20-40 ng/ml) & $24.12(10.5)$ & $27.85(15.6)$ & 0.175 \\
\hline \multicolumn{4}{|l|}{ BMD } \\
\hline Lumbar spine T-Score & $-0.58(1.2)$ & $+0.83(2.5)$ & $0.002 *$ \\
\hline Lumbar spine Z-Score & $-0.12(1.2)$ & $+1.71(2.6)$ & $<0.001 *$ \\
\hline Total femur T-Score & $-1.05(0.9)$ & $-1.10(1.7)$ & 0.886 \\
\hline Total femur Z-Score & $-0.60(0.9)$ & $-0.45(1.7)$ & 0.660 \\
\hline Femoral neck T-Score & $-1.77(1.1)$ & $-1.63(1.6)$ & 0.615 \\
\hline Femoral neck Z-Score & $-0.98(1.1)$ & $-0.55(1.6)$ & 0.189 \\
\hline
\end{tabular}

*Significant differences between patients with 1 or 2 mutations for no/low-trauma fractures. Beyond a specific fracture type and morphology, this may also be reflected in characteristic DXA findings. Accordingly, this data provides first evidence that assessing BMD in HPP may have predictive value in terms of identifying patients with severely compromised mineralization mirrored in pathologically elevated lumbar spine BMD. While patients with pathologically elevated lumbar spine BMD appear to be prone to experience HPP-related fractures, DXA BMD at femoral sites appears to be largely unaffected by the underlying ALPL genotype, making this anatomical region suitable for assessing osteoporosis in patients harboring an ALPL variant, which is particularly important for further strategies in terms of identifying individually adjusted treatment strategies.

This study represents a first step towards discerning patients at risk for HPP-related fractures from patients with increased fracture risk for other reasons carrying an ALPL mutation and it also provides evidence that measuring BMD may

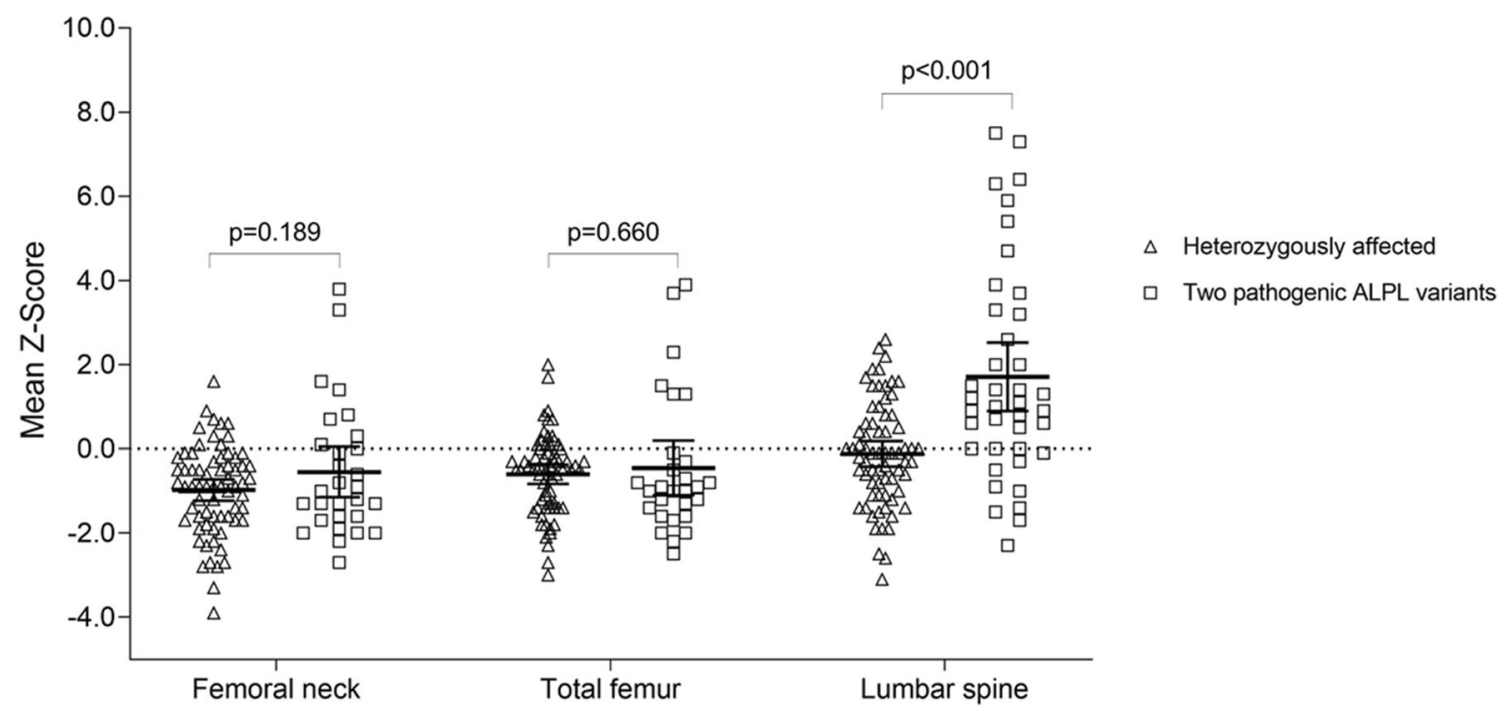

Fig. 3 Differences in femoral and lumbar spine Z-Scores for patients with two pathogenic variants 
Table 3 Characteristics (mean (SD)) for HPP patients with and without HPP-related fractures

\begin{tabular}{|c|c|c|c|}
\hline Mean (SD) & $\begin{array}{l}\text { HPP-related fractures } \\
(\underline{n}=22 / 20 \%)\end{array}$ & $\begin{array}{l}\text { Other patients } \\
(\underline{n}=88 / 80 \%)\end{array}$ & $\underline{p}$ value* \\
\hline \multicolumn{4}{|l|}{ Anthropometrics } \\
\hline Age (year) & $52.73(14.0)$ & $44.57(13.4)$ & $0.013^{*}$ \\
\hline Height $(\mathrm{cm})$ & $167.18(10.6)$ & $167.36(9.7)$ & 0.939 \\
\hline Weight $(\mathrm{kg})$ & $77.05(16.2)$ & $71.93(17.0)$ & 0.205 \\
\hline BMI $\left(\mathrm{kg} / \mathrm{m}^{2}\right)$ & $27.37(4.0)$ & $25.63(5.6)$ & 0.177 \\
\hline \multicolumn{4}{|l|}{ Lab values (normal range) } \\
\hline ALP (f:42-98/m: 53-128 U/l) & $16.68(11.1)$ & $26.32(11.0)$ & $<0.001^{*}$ \\
\hline Phosphate $(0.6-1.8 \mathrm{mmol} / \mathrm{l})$ & $1.58(0.1)$ & $1.30(0.2)$ & $<0.001 *$ \\
\hline PLP (5-30 ng/ml) & $462.78(483.2)$ & $75.33(104.4)$ & $0.001 *$ \\
\hline uPEA (2.3-11.3 mmol/mol Krea) & $59.36(51.9)$ & $16.10(17.3)$ & $0.001 *$ \\
\hline NTX (12.9-22.7 nM BCE/l) & $15.55(13.4)$ & $12.96(4.9)$ & $0.040^{*}$ \\
\hline Vitamin D (20-40 ng/ml) & $28.27(11.6)$ & $24.82(13.8)$ & 0.301 \\
\hline \multicolumn{4}{|l|}{ BMD } \\
\hline Lumbar spine T-Score & $+1.33(2.1)$ & $-0.44(1.7)$ & $<0.001 *$ \\
\hline Lumbar spine Z-Score & $+2.32(2.4)$ & $+0.11(1.7)$ & $<0.001 *$ \\
\hline Total femur T-Score & $-1.36(1.1)$ & $-1.03(1.2)$ & 0.368 \\
\hline Total femur Z-Score & $-0.77(1.4)$ & $-0.53(1.2)$ & 0.527 \\
\hline Femoral neck T-Score & $-1.71(1.0)$ & $-1.74(1.3)$ & 0.943 \\
\hline Femoral neck Z-Score & $-0.72(1.2)$ & $-0.87(1.3)$ & 0.698 \\
\hline
\end{tabular}

*Significant differences between patients with and without HPP-related fractures be an informative tool for routinely assessing disease manifestation in adults with HPP and how to differently interpret associated findings. While patients prone to HPP-associated fractures (increased lumbar spine BMD, previous Pseudofractures, substantially elevated substrate levels, two mutations) may benefit from enhancing ALP enzyme activity in bone and should be spared from treatments binding hydroxyapatite and further reducing bone turnover [30], patients at risk for osteoporotic fractures (reduced femoral BMD, elevated bone turnover markers) without evidence for deficient bone mineralization, treatments aiming at improving BMD may be beneficial to reduce fracture risk. Osteoanabolic strategies that have been probed in that regard include sclerostin-inhibition [31] and solicited individual cases treated with PTH analogues [20, 32,33 ] and even though systematic and long-term evidence for these strategies are lacking, available data and pathophysiologic considerations at least do not imply untoward effects regarding underlying HPP. If and how antiresorptive strategies can elicit a favorable benefit-risk ratio in these patients remains to be scrutinized, specifically when presuming that there may be some residual mineralization deficit and/or low remodeling activity even in the preferentially osteoporotic patients.

The limited number of cases and its retrospective nature can be considered major limitations of this study. Thus, further scientific effort is surely warranted to further refine criteria helping to assign individual patients to specific fracture risk categories and to generate evidence helping to advocate adjusted treatment strategies.

\section{Conclusion}

In adult HPP patients, elevated lumbar spine DXA values appear to be a hallmark of severe disease associated with an increased risk for HPP-related fractures while femoral BMD seems largely unaffected by disease severity. Distinctly analyzing lumbar spine and femoral BMD data may contribute to discriminating HPP-associated fracture risk from other causes of increased fracture risk due to low BMD which does not appear to be affected systematically in subjects with ALPL.

Funding Open Access funding provided by Projekt DEAL.

Data availability All data and study material is stored at the Department of Orthopedics at the University of Wuerzburg for 10 years. The datasets used and analyzed during the current study are available from the corresponding author on reasonable request.

\section{Compliance with ethical standards}

Conflicts of interest FG reports personal fees from Abbvie, Alexion, and Lilly. LS reports personal fees from Abbvie, Amgen, Alexion, KyowaKirin, Lilly, Medi, MSD, Novartis, Servier, and UCB and research grants to the Institution (University of Wuerzburg) from Alexion, Kyowa Kirin, and Novartis. LC and DR have nothing to disclose during the study course.

Ethics approval The study protocol was approved by the competent ethics committee at Wuerzburg University (Nr. 20180105 01). 
Consent to participate/publication n.a. (retrospective chart review)

Open Access This article is licensed under a Creative Commons Attribution-NonCommercial 4.0 International License, which permits any non-commercial use, sharing, adaptation, distribution and reproduction in any medium or format, as long as you give appropriate credit to the original author(s) and the source, provide a link to the Creative Commons licence, and indicate if changes were made. The images or other third party material in this article are included in the article's Creative Commons licence, unless indicated otherwise in a credit line to the material. If material is not included in the article's Creative Commons licence and your intended use is not permitted by statutory regulation or exceeds the permitted use, you will need to obtain permission directly from the copyright holder. To view a copy of this licence, visit http://creativecommons.org/licenses/by-nc/4.0/.

\section{References}

1. Whyte MP (2016) Hypophosphatasia - aetiology, nosology, pathogenesis, diagnosis and treatment. Nat Rev Endocrinol 12(4):233246

2. Mornet E (2018) Hypophosphatasia. Metabolism 82:142-155

3. Hofmann $\mathrm{C}$ et al (2014) Unexpected high intrafamilial phenotypic variability observed in hypophosphatasia. Eur J Hum Genet 22(10): 1160-1164

4. Berkseth KE et al (2013) Clinical spectrum of hypophosphatasia diagnosed in adults. Bone 54(1):21-27

5. Whyte MP, Wenkert D, Zhang F (2016) Hypophosphatasia: natural history study of 101 affected children investigated at one research center. Bone 93:125-138

6. Whyte MP et al (2015) Hypophosphatasia: validation and expansion of the clinical nosology for children from 25 years experience with 173 pediatric patients. Bone 75:229-239

7. Millan JL, Whyte MP (2016) alkaline phosphatase and hypophosphatasia. Calcif Tissue Int 98(4):398-416

8. McKiernan FE, Berg RL, Fuehrer J (2014) Clinical and radiographic findings in adults with persistent hypophosphatasemia. J Bone Miner Res 29(7):1651-1660

9. Lopez-Delgado L et al (2018) Abnormal bone turnover in individuals with low serum alkaline phosphatase. Osteoporos Int

10. Anderson HC et al (2005) Sustained osteomalacia of long bones despite major improvement in other hypophosphatasia-related mineral deficits in tissue nonspecific alkaline phosphatase/nucleotide pyrophosphatase phosphodiesterase 1 double-deficient mice. Am J Pathol 166(6):1711-1720

11. Rassie K et al (2019) Bisphosphonate use and fractures in adults with hypophosphatasia. JBMR Plus 3(10):e10223

12. Alonso N et al (2019) Loss-of-function mutations in the ALPL gene presenting with adult onset osteoporosis and low serum concentrations of total alkaline phosphatase. J Bone Miner Res

13. Jain RK, Vokes T (2017) Dual-energy X-ray absorptiometry. J Clin Densitom 20(3):291-303

14. Lewiecki EM (2010) Bone densitometry and vertebral fracture assessment. Curr Osteoporos Rep 8(3):123-130
15. Girschick HJ et al (2007) Long-term follow-up of bone mineral density in childhood hypophosphatasia. Joint Bone Spine 74(3): 263-269

16. Schmidt $\mathrm{T}$ et al (2017) Clinical, radiographic and biochemical characteristics of adult hypophosphatasia. Osteoporos Int 28(9):26532662

17. Genest F, Seefried L (2018) Subtrochanteric and diaphyseal femoral fractures in hypophosphatasia-not atypical at all. Osteoporos Int

18. Whyte MP (2009) Atypical femoral fractures, bisphosphonates, and adult hypophosphatasia. J Bone Miner Res 24(6):1132-1134

19. Sutton RA et al (2012) "Atypical femoral fractures" during bisphosphonate exposure in adult hypophosphatasia. J Bone Miner Res 27(5):987-994

20. Whyte MP, Mumm S, Deal C (2007) Adult hypophosphatasia treated with teriparatide. J Clin Endocrinol Metab 92(4):1203-1208

21. Alt V et al (2013) A new metaphyseal bone defect model in osteoporotic rats to study biomaterials for the enhancement of bone healing in osteoporotic fractures. Acta Biomater 9(6):7035-7042

22. Kherad $\mathrm{M}$ et al (2015) The number and characteristics of prevalent vertebral fractures in elderly men are associated with low bone mass and osteoporosis. Bone Joint J 97-b(8):1106-1110

23. Tadokoro $\mathrm{M}$ et al (2009) New bone formation by allogeneic mesenchymal stem cell transplantation in a patient with perinatal hypophosphatasia. J Pediatr 154(6):924-930

24. Chuck AJ et al (1989) Crystal deposition in hypophosphatasia: a reappraisal. Ann Rheum Dis 48(7):571

25. Lassere MN, Jones JG (1990) Recurrent calcific periarthritis, erosive osteoarthritis and hypophosphatasia: a family study. J Rheumatol 17(9):1244-1248

26. Whyte MP (2017) Hypophosphatasia: an overview For 2017. Bone 102:15-25

27. Nordin BEC et al (1996) Regional bone mineral density interrelationships in normal and osteoporotic postmenopausal women. J Bone Miner Res 11(6):849-856

28. Whyte MP, Murphy WA, Fallon MD (1982) Adult hypophosphatasia with chondrocalcinosis and arthropathy. Variable penetrance of hypophosphatasemia in a large Oklahoma kindred. Am J Med 72(4):631-641

29. Guañabens N et al (2014) Calcific periarthritis as the only cclinical manifestation of hypophosphatasia in middle-aged sisters. J Bone Miner Res 29(4):929-934

30. Drake MT, Clarke BL, Khosla S (2008) Bisphosphonates: mechanism of action and role in clinical practice. Mayo Clin Proc 83(9): 1032-1045

31. Seefried L et al (2017) Efficacy of anti-sclerostin monoclonal antibody BPS804 in adult patients with hypophosphatasia. J Clin Invest 127(6):2148-2158

32. Camacho PM et al (2016) Adult hypophosphatasia treated with teriparatide: report of two patients and review of the literature. Endocr Pract

33. Schalin-Jantti C et al (2010) Parathyroid hormone treatment improves pain and fracture healing in adult hypophosphatasia. J Clin Endocrinol Metab 95(12):5174-5179

Publisher's note Springer Nature remains neutral with regard to jurisdictional claims in published maps and institutional affiliations. 